\title{
A case of young infant HPeV1 infection with intraventricular hemorrhage
}

Kiyohito Okumiya, Kenji Gotoh, Kazuaki Tatara, Yousuke Nakajima, Mariko Teramachi, Yushiro Yamasita

The Department of Pediatrics and Child Health Kurume University School of Medicine, Fukuoka, Japan

\section{There is no conflict of interest in this study.}

【Back ground】

Human parechoviruses (HPeVs) are RNA

viruses that are classified in the family

Picornaviridae and 17 genotypes are

confirmed. In Europian study HPeV was

detected in $3-8 \%$ of children presenting to

emergency with undifferentiated fever and in

children requiring hospital admission, where

up to $15-20 \%$ of children with fever without

source are HPeV-positive. Serological data

from Japan show that $90 \%$ of infants have

been infected with at least one HPeV subtype

by the age of 2 years. HPeVs usually cause

mild respiratory or gastrointestinal symptoms, but $\mathrm{HPeV} 1$ and $\mathrm{HPeV} 3$ are known that they can provoke sepsis and meningoencephalitis leading to neurological sequelae in neonates and young infants. Here we report a case of $\mathrm{HPeV} 1$ infection with Intraventricular hemorrhage.

\section{【Case】}

Age: 2 months, Sex: male

$<$ Perinatal history>

Gestational age:36w0d

Birth weight 2388g, MD twin

$<$ CC>

Fever, Scarlet Skin, irritable

<History of present illness>

$X$-2days Poor suckling, irritable

$X-1$ day Fever (admitted in local hospital)

X-day Transferred Kurume University

<sick contact> Hospital due to sepsis.

Not in particular.

$<$ Vaccination $>$

Hib $\times 1$, PCV $\times 1$

Copyright $\odot 2018$ Kiyohito Okumiya, Kenji Gotol The Department of Pediatrics and Child Hea Kurume University School of Medicin
$<$ Physical finding $>$

HT: $60 \mathrm{~cm}(+1.0 \mathrm{SD})$, BW: $6.1 \mathrm{~kg}(+0.9 \mathrm{SD})$

BT: $38.1^{\circ} \mathrm{C}, H R: 205 \mathrm{bpm}, \mathrm{BP}: 100 / 70 \mathrm{mmHg}$,

RR: 44/min, SpO2: $98 \%$

General condition: not doing well

irritability $(+)$

Head: fontanelle open flat, Neck stiffness(+)

Throat: slightly injected,

LNS: not palpable,

Chest: no rale, regular, no murmur, III(-)

Abdomen: soft, mass(-), BS normal

Skin: rash on face and perineum

Pupils: $3 \mathrm{~mm} / 3 \mathrm{~mm}$, reflex $+/+$,

Nystgmus:(-)

Babinski -/- Chaddock -/-

$\langle$ Blood gas〉

$\mathrm{pH} \quad 7.400$

pCO2 $32.7 \mathrm{mmHg}$ Tbil $0.9 \mathrm{mg} / \mathrm{dl}$

HCO3 $19.8 \mathrm{mmol} / \mathrm{l} \quad$ AST $976 \mathrm{IU} / \mathrm{l}$

$\begin{array}{lllll}\text { B.E } & 3.8 & \text { ALT } & 517 & \mathrm{IU} / \mathrm{l}\end{array}$

Lac $1.9 \mathrm{mmol} / \mathrm{l} \quad \mathrm{LDH} 2387 \mathrm{IU} / \mathrm{I}$

CK 2167 IU/I

〈Coagulability〉 CK-MB 194 IU/I

APTT $44.5 \mathrm{sec}$ CRP $2.3 \mathrm{mg} / \mathrm{dl}$

PT(ACT) $48 \% \quad$ PCT $28.6 \mathrm{ng} / \mathrm{dl}$

$\begin{array}{llll}\mathrm{PT}(\mathrm{INR}) & 1.49 & \mathrm{Na} & 142 \mathrm{mEq} / \mathrm{L}\end{array}$

FDP $\quad 49 \mathrm{mg} / \mathrm{dl} \quad \mathrm{K} \quad 5.0 \mathrm{mEq} / \mathrm{L}$

AT $\quad 59 \% \quad \mathrm{Cl} \quad 112 \mathrm{mEq} / \mathrm{L}$

$\mathrm{Ca} \quad 9.5 \mathrm{mg} / \mathrm{dl}$

〈CBC〉 BUN $8.5 \mathrm{mg} / \mathrm{dl}$

WBC $12200 / \mu \mathrm{l} \quad$ Cre $0.26 \mathrm{mg} / \mathrm{d}$

Neut $25 \% \quad$ Glu $91 \mathrm{mg} / \mathrm{dl}$

Lymph $\quad 71 \% \quad$ Ferritin $212 \quad \mathrm{ng} / \mathrm{dl}$

RBC $35810^{\wedge} 4 / \mu \mathrm{l} \quad$ IL2R $2074 \quad \mathrm{U} / \mathrm{ml}$

$\mathrm{Hb} \quad 9.7 \mathrm{~g} / \mathrm{dl} \quad$ PIVKA II $37 \mathrm{mAU} / \mathrm{ml}$

PIt $34.010^{\wedge} 4 / \mu \mathrm{l} \quad$ proBNP $5522 \mathrm{pg} / \mathrm{ml}$

〈Chest X-ray〉 CTR: $56 \%$, no abnomal findings

$\langle$ Heart US〉 EF:70\%,

no valvular regurgitation, no pericardial effusion $\langle$ Figure 1〉 Clinical course

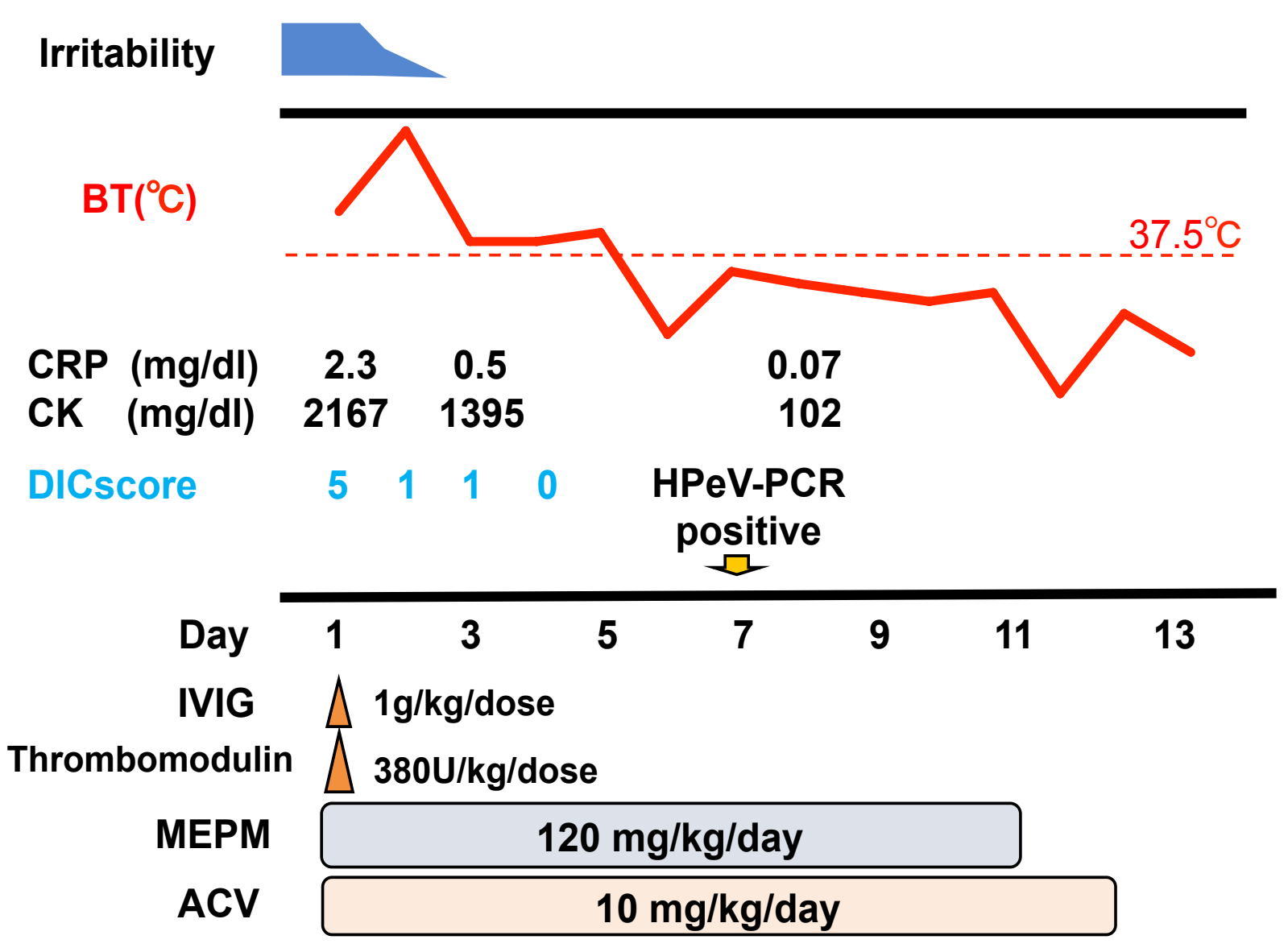

$\langle$ Viral genetics test $\rangle$

Samples:

blood, spinal fluid, throat swab, urine, feces

Results :

Parvovirus B-19 : negative

Adenovirus : negative

Echovirus: negative

Herpes simplex virus: negative

Cytomegalovirus: negative

Enterovirus (universal): positive (blood, feces)

Human parechoviruses 1 : positive (blood, feces)

$\langle$ Clinical course〉 (Figure 1)

Meropenem and aciclovir were prescribed for empiric treatment of sepsis caused by unknown and thrombomodulin was combined for DIC. He became afebrile on day 5 and his systemic condition was improved. In a head CT on day 4 , Intraventricular hemorrhage was found at posterior horn of lateral ventricle. (Figure 2)

With conservative treatment, hemorrhage was disappearing on day 11. HPeV1 was detected by blood and fecal PCR and it was thought that a series of symptoms were caused by viremia of HPeV1. There are no previous neurologic sequelae at hospital discharge.
〈Figure 2〉 Head CT

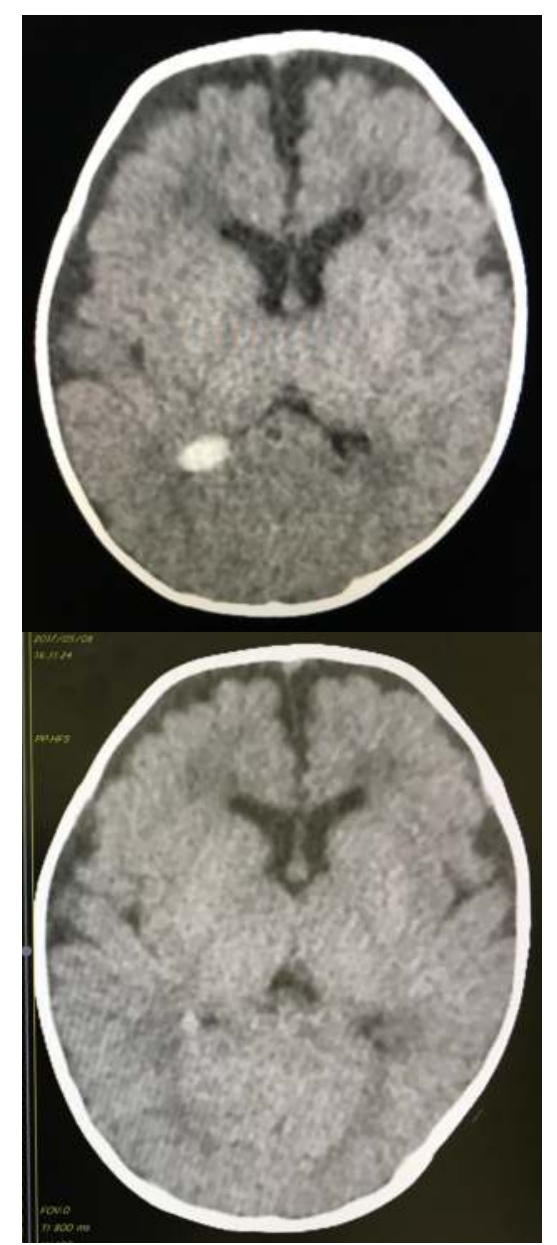

Day 4

【Discussion】

$\mathrm{HPeVs}$ infections are most commonly associated with mild gastrointestinal or respiratory symptoms $\mathrm{HPeV} 3$ has been associated with more-severe disease, such as neonatal sepsis and meningitis. I1 Compared with HPeV3, HPeV1 symptoms are relatively mild and there are few CNS symptoms. There is no report of HPeV1 that caused cerebral hemorrhage so far. This case is important that it can merge intraventricular hemorrhage even with HPeV1 infection. [2]

This case had clinical symptoms of sepsis with evaluation of DIC score and met criteria for SIRS. It seemed that vascular endothelial damage was caused due to cytokine storm, therefore LDH and IL2R, PCT were elevated, and might be relevant to the Intraventricular haemorrhage. [3]

【Take home message】

In case of young infant with clinical symptoms of sepsis, it is necessary to rule out HPeVs infection. And consideration the possibility of cerebral hemorrhage is important not only $\mathrm{HPeV} 3$ infection but HPeV1.

[References】

1. Wolthers KC, et al: Clin Infect Dis 47:358-363, 2008 2. Eur J Pediatr. 2015 Dec;174(12):1639-47.Epub 2015 Jun 25 3. Fortuna, et al: J Clin Virol. 2017 Jan;86:39-45 